\title{
Polarization Dynamics of VDF-TrFE Copolymers
}

\author{
G. Eberle, E. Bihler and \\ W. Eisenmenger, \\ Physikalisches Institut, Universität Stuttgart, \\ Stuttgart, Germany
}

\begin{abstract}
The polymer PVDF and its copolymers with TrFE show a strong piezoelectric effect after poling in high electric fields $(100 \mathrm{MV} / \mathrm{m})$. In order to study the dynamics of the poling process we have applied HV impulses of definite duration to the polymer. The dielectric displacement during the $\mathrm{HV}$ impulse is recorded. After the impulse the remanent polarization under short circuit condition is measured. Thus it is possible to obtain the minimum poling time dependent on the applied field strength necessary to stabilize the remanent polarization. By comparing the time development of the dielectric displacement with the corresponding remanent polarization we find a time delay between the orientation of the dipoles and their stabilization. Therefore we conclude that the process of orientation of the dipoles itself is not sufficient to lead to a remanent polarization. An additional interaction between trapped charges and the oriented dipoles can explain the stability of the remanent polarization and the observed time delay.
\end{abstract}

\section{INTRODUCTION}

$K_{\text {AWAI discovered piezoelectricity in the polymer of }}$ $I_{\text {polyvinylidenefluoride (PVDF) in 1969. Later it was }}$ shown that the copolymers of vinylidenefluoride (VDF) with trifluoroethylene ( $\operatorname{TrFE}$ ) also exhibit piezoelectricity [2].

In the last twenty years significant progress has been made to explain piezoelectricity in both polymers [35]. Today it is commonly accepted that the spontaneous and remanent polarization of the crystallites of the polar $\beta$-phase is responsible for the high piezoelectric constants in both polymers. After applying high electric fields $(\sim 100 \mathrm{MV} / \mathrm{m})$ to thin films of PVDF and P(VDF/
TrFE) the crystallite dipoles can be permanently aligned. Unpoled films show no piezoelectricity. The piezoelectric response can be described mainly by a change in the number density of aligned crystallite dipole moments under strain. While PVDF crystallizes in the unpolar $\alpha$-phase, the $\mathrm{P}$ (VDF/TrFE) copolymer crystallizes directly in the $\beta$-phase, if the TrFE portion is greater than 15 mole\% [6]. $\alpha$-PVDF is transformed to the polar $\beta$-phase by stretching the films.

The fact that the crystallite dipoles are permanently aligned by an external electric field indicates that PVDF and $\mathrm{P}(\mathrm{VDF} / \mathrm{TrFE})$ are 'hard' (by analogy with ferromagnetic materials) ferroelectric polymers. The hard ferroelectric behavior has been attributed to the anisotropy 
field of the near-hexagonal lattice of the $\beta$-phase crystallites [8]. This model predicts a switching of the dipoles under field in steps of $60^{\circ}$ from one potential minimum to another [7]. If all dipoles of a crystallite are aligned into the field direction they would remain in this direction after turning off the electric field and the polarization would be immediately remanent. Despite the success of the $60^{\circ}$ model to explain the orientation of dipoles under field some experimental observations are still difficult to interpret within the frame of this model:

By poling with lower field strength spatial inhomogeneous polarization distributions are found in PVDF. The investigation of the development of these inhomogeneous distributions during the poling process gives strong evidence that injection and migration of space charges determine the polarization distribution [9].

Another point is the surprising stability of the remanent polarization in both polymers at room temperature for many years. In addition, the coercitive field strength is three orders of magnitude larger than in other ferroelectrics [3].

Based on the switching experiments of Merz in $\mathrm{BaTiO}_{3}$ single crystals [10] similar experiments were carried out with PVDF [11] and $\mathrm{P}(\mathrm{VDF} / \mathrm{TrFE})[12-15]$ to compare with the predictions of the $60^{\circ}$ model. The switching times derived from the dielectric displacement under field agree with the switching times predicted by the $60^{\circ}$ model [24]. In all these experiments it has been tacitly assumed that the remanent polarization is immediately stable for short-time field application and the dipoles remain oriented in the field directions after removing the electric field. Thus in the case of PVDF as a hard ferroelectric material with intrinsic potential walls, it is expected that the dielectric displacement $D$ and the remanent polarization $P_{\text {rem }}$ show the same time constants during the poling procedure.

Figure 1 shows this expectation schematically for unpoled films: An external electric field is applied at $t=0$ to unpoled PVDF or $\mathrm{P}(\mathrm{VDF} / \mathrm{TrFE})$ films. There is a fast polarization attributed to electronic and ionic displacement. The orientation polarization starts with some delay at $t=t_{0}$.

At $t_{1}$ all dipoles are aligned into the field direction and the displacement reaches its maximum. At longer poling times the displacement will not grow any more. If the electric field is switched off at a time between $t_{0}$ and $t_{1}$ the partly oriented crystallite dipoles will remain in the field direction, resulting in the corresponding remanent polarization $P_{\text {rem }}$. It is expected that both the remanent

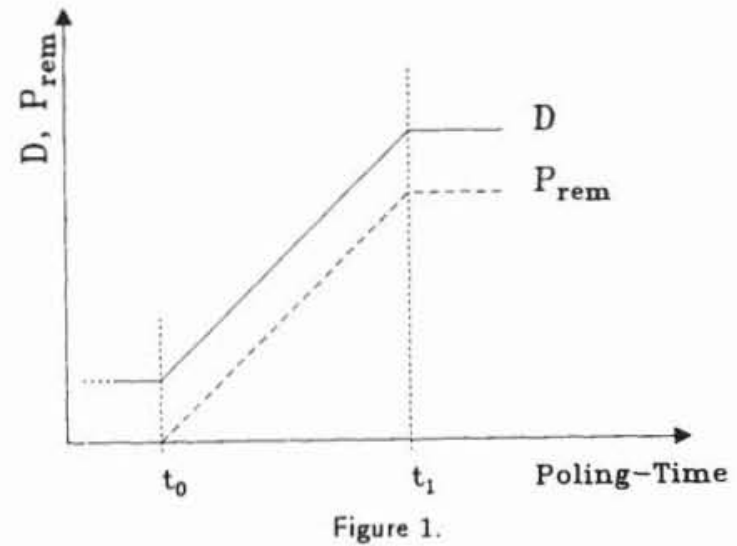

Schematic diagram for the development of the dielectric displacement $D$ and the remanent polarization $P_{\text {rem }}$ with poling time $t$. The electric field is switched on at $t=0$. The orientation of dipoles starts at $t=t_{0}$. If dipoles come into a new equilibrium position as expected by the $60^{\circ}$ model they stay in that position and contribute to the remanent polarization $P_{\text {rem }}$.

polarization and the displacement reach their maximum at $t_{1}$ (Figure 1).

In Figure 2 the expected behavior is shown for the case of polarization reversal of prepolarized films. At $t_{0}$ the dipoles begin to invert under the field and the displacement starts to grow as in the case of unpoled films (compare Figure 1). At $t_{1}$ all dipoles are inverted under the field and the displacement attains its maximum. For longer poling times it remains constant. Under short circuit the remanent polarization in the original direction first decreases to zero and then grows in the new, opposite direction. The remanent polarization in the opposite direction saturates again at $t_{1}$.

Since the values of the remanent polarization are the same (but in opposite direction) before the application of HV $\left(P_{\text {rem,pre }}\right)$ and after saturation $\left(P_{\text {rem,inv }}\right)$, the reversed polarization will cross zero value, when the displacement $D$ has reached half of its final saturation value. In the experiments we will compare this time $t_{x}$ for the remanent polarization to cross zero value with the corresponding time $t_{2}$ for the displacement to reach half of the saturation value. In the expectation of the $60^{\circ}$ model however, these times $t_{1}$ and $t_{2}$ should coincide, since the dipoles are immediately stabilized in their new positions.

In contrast to these expectations, our measurements show a significant time delay between the development 


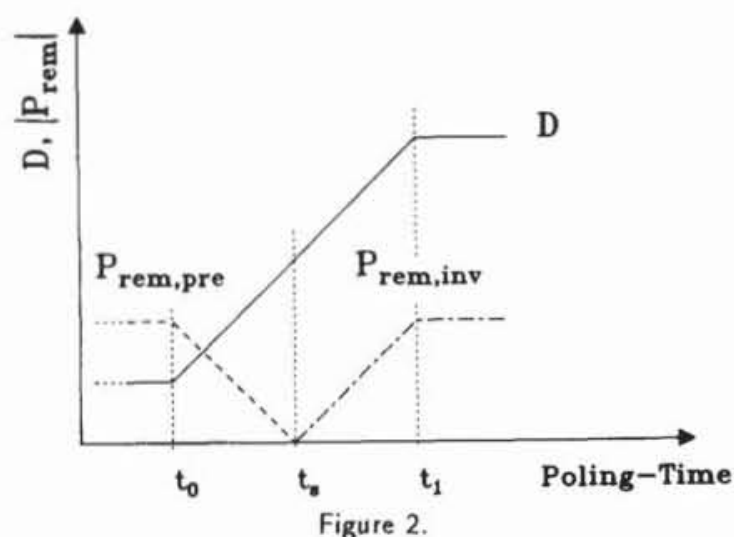

Schematic diagram for the development of the dielectric displacement $D$ and the remanent polarization $P_{\text {rem }}$ with poling time $t$ in the case of polarization reversal.

of the remanent polarization and the immediate fast development of the displacement by poling and inverting the remanent polarization of as-received PVDF films with lower $(37 \%)$ and higher $(85 \%) \beta$-content [16]. These measurements indicate that the dipole orientation under field and the development of the remanent polarization are due to different mechanisms with correspondingly different time constants.

This raises the questions: Does a corresponding time delay also show up in $\mathrm{P}(\mathrm{VDF} / \mathrm{TrFE})$ copolymers? Is the stabilization process of the remanent polarization also consistent with a model where charges are trapped at the boundaries of the crystallites $[18,16,23]$ explaining the time delay as measured in PVDF? In the following we present some corresponding results.

\section{EXPERIMENTAL}

$\mathrm{F}_{\mathrm{s}}^{\mathrm{om}}$ OR the experiments a specially developed HV pulse generator has been used as described in [16]. Figure 3 shows the block diagram of the circuit: High voltage is applied to the sample by opening switch S2 and closing switch S1. After charging for a definite pulse length, the sample is later shorted by opening S1 and closing S2. Both switches consist of HV field effect transistors triggered by external pulse generators.

The dielectric displacement $D$ is measured via the voltage $U_{c}$ at capacitor $C$, which capacitance is large compared to the sample capacitance:

$$
D=\frac{Q}{A}=\frac{U_{c} * C}{A}
$$

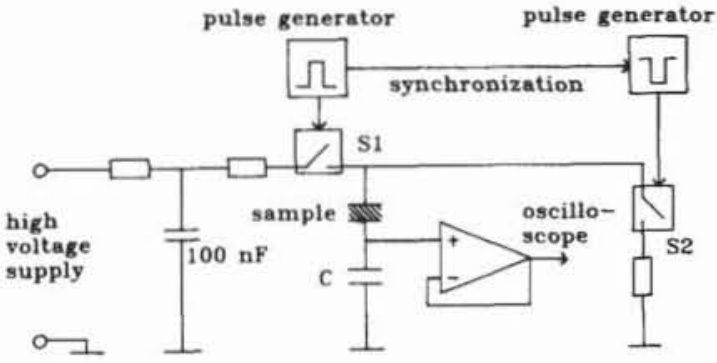

Figure 3.

Block diagram of the HV pulse generator used for poling the samples.

where $A=15.6 \mathrm{~mm}^{2}$ is the sample area, and $C=$ $50 \ldots 300 \mathrm{nF}$.

The time dependent voltage $U_{c}$ is recorded by a digital storage oscilloscope. The dielectric displacement was always calculated at the end of $\mathrm{HV}$ pulse. Following the $\mathrm{HV}$ pulse the sample was kept for several minutes under short circuit conditions. After this the remanent polarization is measured with the PPS-method (Piezoelectrically generated Pressure Step) $[17,18]$. Thus the dielectric displacement and the remanent polarization can be measured simultaneously for one individual sample for a definite poling time. For each poling time we used a new sample. Therefore in Figures 4 and 5 each measured point corresponds to a different sample. The remanent polarization can also be obtained from the voltage at capacitor $C$. But the capacitor gives only a spatial average. Therefore we used the PPS-technique for the measurement of the polarization distribution and also of its sign.

The $75 / 25$ mole\% $\mathrm{P}$ (VDF/TrFE) samples consist of 75 mole $\%$ VDF and 25 mole\% TrFE. The films of $(12 \pm 5$ $\mu \mathrm{m}$ thickness were cast from methylethylketone solution at room temperature and then annealed at $120^{\circ} \mathrm{C}$ for $2 \mathrm{~h}$. The influence of the annealing temperature is reported in the literature $[15,20-22]$. The crystallinity of the films is increased. With higher annealing temperature we find a reduction of the poling time necessary to achieve saturation.

\section{RESULTS}

\subsection{UNPOLARIZED FILMS}

$\mathrm{F}$ GURE 4 shows the dielectric displacement $D$ and the F remanent polarization $P_{\text {rem }}$ as functions of the pulse length for poling at various field strengths $E$. The start time of the development of the displacement and the remanent polarization defined by the linear extrapolation 

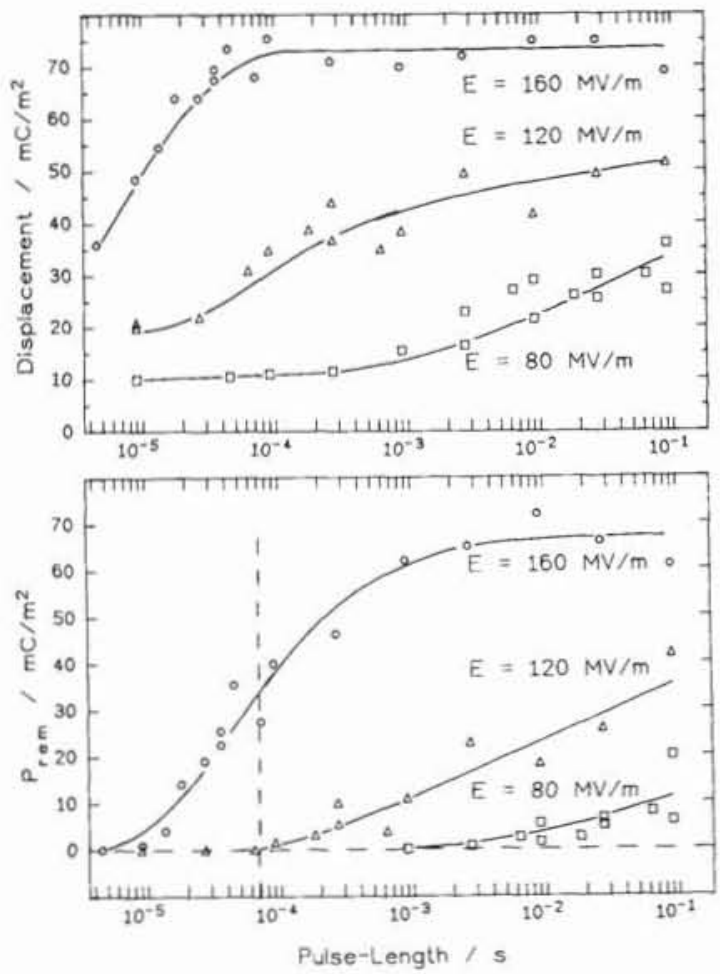

Figure 4.

Time development of the displacement (top) and the remanent polarization (bottom) dependent on the applied HV pulse length. Unpoled films of $75 / 25$ mole\% P(VDF/TrFE) copolymer. Film thickness $(12 \pm 5 \mu \mathrm{m})$. Applied field strength: ㅁ: $80 \mathrm{MV} / \mathrm{m} ; \Delta: 120 \mathrm{MV} / \mathrm{m} ;$ o: $160 \mathrm{MV} / \mathrm{m}$. The vertical line marks the time, when half of the saturation value is reached with $160 \mathrm{MV} / \mathrm{cm}$.

of the maximum slope to zero depends on the applied field strength. In order to compare the time development of the displacement with the remanent polarization, the time, when half of the final value is reached can be taken from the Figures. These times are listed in Table 1.

The remanent polarization starts to grow later and increases more slowly than the displacement. There is a difference of approximately one order of magnitude in time between the development of the displacement and the remanent polarization. This is independent from the reduction of the time scales with increased field. Doubling the field strength reduces the times by a factor of approximately three orders of magnitude in time.

Compared with corresponding measurements with pure
PVDF films [16], the polarization development in the copolymer films is more than two orders of magnitude faster. The time scale is determined by the crystallinity and the $\beta$ content. With increased crystallinity and $\beta$ content the poling times are reduced.

\subsection{POLARIZATION REVERSAL}

As with PVDF films, the dynamics of polarization reversal in copolymer films are of fundamental and also practical relevance. The samples were poled for $100 \mathrm{~s}$ using the same field strength as later applied in opposite direction in order to reverse the polarization. One exception: Samples to be reversed with $160 \mathrm{MV} / \mathrm{m}$ field strength were poled with $200 \mathrm{MV} / \mathrm{m}$. After poling all samples were kept under short circuit condition for another $100 \mathrm{~s}$. After this procedure the samples had a remanent polarization (see Table 2)

Table 2.

\begin{tabular}{|c|c|}
\hline \hline Field MV $/ \mathrm{m}$ & Polarization $\mathrm{mC} / \mathrm{m}^{2}$ \\
\hline 200 & $105 \pm 10 \%$ \\
120 & $68 \pm 10 \%$ \\
80 & $48 \pm 10 \%$ \\
\hline
\end{tabular}

Subsequently to this a HV impulse of definite duration was applied to the samples in opposite direction in order to reverse the polarization.

The displacement was measured with the capacitor $C$ in series with the sample. The samples are kept under short circuit condition for a few minutes before the remanent polarization was measured using the PPS method. It is a great advantage that the PPS method reveals also the sign of the remanent polarization.

Figure 5 shows the displacement and the remanent polarization as a function of the pulse length at different field strengths. Again a strong field dependence is to be seen. At $80 \mathrm{MV} / \mathrm{m}$ the displacement remains at a low level of $10 \mathrm{mC} / \mathrm{m}^{2}$ until $300 \mu$ s pulse length. For a longer pulse duration it increases until $5 \mathrm{~ms}$ pulse length and finally saturates. The amount of remanent polarization decreases after $100 \mu$ s pulse length, but it is not inverted before $2.5 \mathrm{~ms}$. After $2.5 \mathrm{~ms}$ it starts to increase in the opposite direction and saturates after $100 \mathrm{~ms}$. In this time interval the displacement has already saturated.

It is remarkable that the inverted remanent polarization raises with about the same delay as observed in the 
Table 1.

Comparison of typical times for the development of displacement and remanent polarization in $\mathrm{P}(\mathrm{VDF} / \mathrm{TrFE})$ copolymer (taken from Figure 4) and pure PVDF [16].

\begin{tabular}{|c|c|c|c|c|}
\hline $\begin{array}{c}\text { field } \\
\text { strength } \\
\mathrm{MV} / \mathrm{m}\end{array}$ & $\begin{array}{c}\text { start of the development } \\
\text { displacement } \\
\mu \mathrm{s}\end{array}$ & $\begin{array}{c}\text { remanent Pol. } \\
\mu \mathrm{s}\end{array}$ & $\begin{array}{c}\text { half of final values reached } \\
\text { displacement } \\
\mu \mathrm{s}\end{array}$ & $\begin{array}{c}\text { remanent Pol. } \\
\mu \mathrm{s}\end{array}$ \\
\hline $\begin{array}{c}\mathrm{P}(\mathrm{VDF} / \mathrm{TrFE}): \\
80\end{array}$ & 400 & 3000 & 1000 & 17000 \\
120 & 30 & 100 & 50 & 2000 \\
160 & 1 & 10 & $<10$ & 80 \\
$\begin{array}{c}\text { pure PVDF: } \\
200\end{array}$ & $<10$ & 80 & 10 & 3000 \\
120 & 200 & 2000 & 20000 & 40000 \\
\hline \hline
\end{tabular}
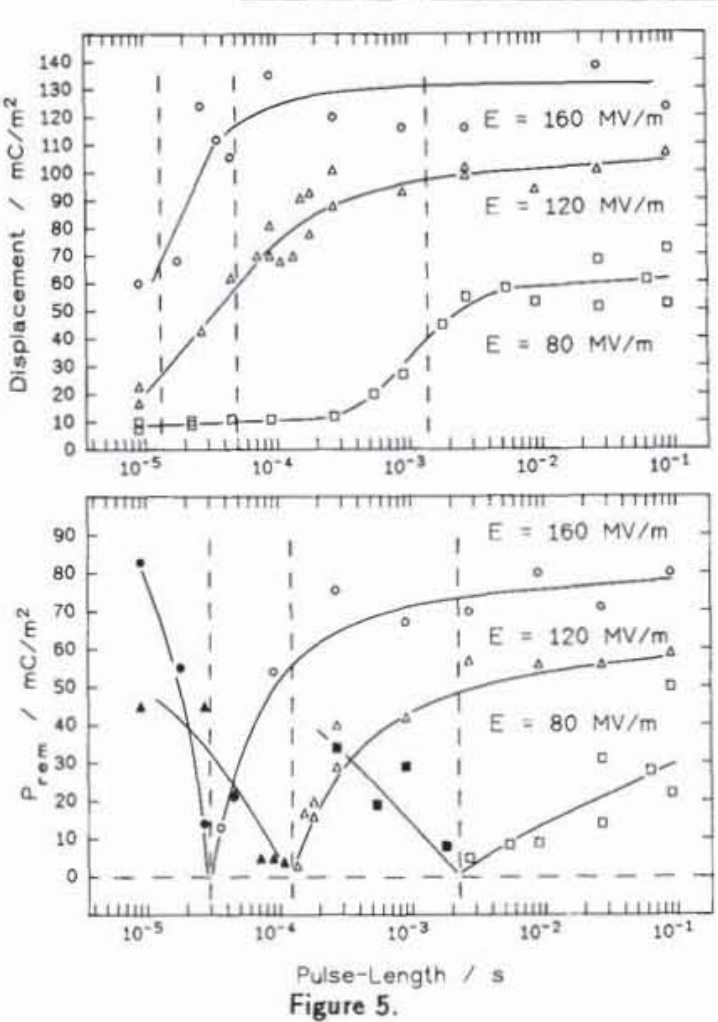

Polarization reversal: Time development of the displacement (top) and the remanent polarization (bottom) dependent on the applied HV pulse length. Prepolarized films of $75 / 25$ mole\% P(VDF/TrFE) copolymer. Film thickness ( $12 \pm 5$ $\mu \mathrm{m})$. Applied field strength: $\square: 80 \mathrm{MV} / \mathrm{m} ; \triangle$ : $120 \mathrm{MV} / \mathrm{m}$; o: $160 \mathrm{MV} / \mathrm{m}$. The vertical lines mark the time $t_{2}$ when the displacement reaches half of its saturation value.

poling of unpolarized samples (Figure 4). Therefore the stabilization of the dipoles in the new direction is caused by the same mechanism in poling unpolarized samples as well as in poling polarized samples. After inversion of its sign the remanent polarization grows faster as compared to unpolarized samples.

Table 3 compares the time $t_{\mathbf{d}}$, when the remanent polarization crosses zero value, with the time $t_{2}$, when the displacement reaches half of its final value.

Table 3.

Comparison of the time development: $t$, pulse length necessary to reverse the remanent polarization to zero value; $t_{2}$ poling time to reach half of the saturation displacement under field.

\begin{tabular}{|c|c|c|}
\hline \hline field strength MV $/ \mathrm{m}$ & $t_{2}$ & $t_{t}$ \\
\hline 80 & $1.2 \mathrm{~ms}$ & $2.5 \mathrm{~ms}$ \\
120 & $50 \mu \mathrm{s}$ & $130 \mu \mathrm{s}$ \\
160 & $15 \mu \mathrm{s}$ & $30 \mu \mathrm{s}$ \\
\hline \hline
\end{tabular}

Thus the observed time delay between the development of the dipole orientation and the stabilization of this orientation is in contrast to the expectation deduced from the cooperative $60^{\circ}$ model. In view of this model it would be natural that the orientation of dipoles under field and their stabilization after removing the external field show no significant time difference. Therefore both times $t_{2}$ and $t_{\mathrm{s}}$ should coincide.

Previous experiments with pure PVDF films [16] show that the corresponding times are more than two orders of magnitude larger. At $120 \mathrm{MV} / \mathrm{m}$ field strength we measured time $t_{\mathrm{s}}=15 \mathrm{~ms}$ for PVDF films with a $\beta$ content of $85 \%$. 


\subsection{POLING OF UNPOLARIZED SAMPLES WITH REPEATED SHORT PULSES}

Using periodically repeated pulses for sample poling it is possible to observe the time dependent development of the displacement and of the remanent polarization in one sample. This reduces the strong scattering of data points by using a new sample for each poling procedure.

In this experiment poling field strength was again 80 $\mathrm{MV} / \mathrm{m}$. Under this field strength the poling process takes place in a few seconds and it is possible to measure the displacement as well as the remanent polarization using the PPS-method. HV is applied to the sample by a thin metalized PETP film. The PPS-signals are observed on the oscilloscope screen and recorded with a video system. Applying HV pulses with $100 \mathrm{~ms}$ pulse length to the sample and shorting between the pulses allows the simultaneous observation of the displacement and the remanent polarization in steps of e.g. $100 \mathrm{~ms}$ poling time. The pulse length of $100 \mathrm{~ms}$ was short enough that after the first pulse the sample is not completely poled and that we can analyze each HV pulse. (The video system needs $20 \mathrm{~ms}$ for one picture!) The displacement is determined at the end of the pulses and the remanent polarization before the beginning of the next pulse. This remanent polarization is larger than the long-time remanent polarization as determined in the single pulse experiments discussed before. But it can also be used as a qualitative measure for the long time remanent polarization.

Samples were $15 \pm 5 \mu \mathrm{m}$ thick $75 / 25$ mole\% $\mathrm{P}$ (VDF/ $\operatorname{TrFE}$ ) films annealed at $135^{\circ} \mathrm{C}$ for $2 \mathrm{~h}$.

In this experiment 100 pulses were applied to every sample. The short circuit time $t_{s c}$ between the pulses varied from $30 \mathrm{~ms}$ to $100 \mathrm{~s}$. Figures 6 and 7 show the results. Figure 6 represents the displacement $D_{n}$ at the $n$-th pulse related to the displacement at the 100 th pulse $D_{n} / D_{100}$. Figure 7 represents the remanent polarization after the $n$-th pulse $P_{n}$ related to the remanent polarization after the 100th pulse $P_{n} / P_{100}$. Each pulse corresponds to a poling time of $100 \mathrm{~ms}$.

The values were related to the last pulse because the absolute values differed from sample to sample. After the first pulse the displacement took a value between 30 and $40 \mathrm{mC} / \mathrm{m}^{2}$, the remanent polarization between 10 and 25 $\mathrm{mC} / \mathrm{m}^{2}$.

Figures 6 and 7 show that there is no change in the time development of both the displacement and the remanent polarization, if $t_{s c}$ lasts $\leqslant 1 \mathrm{~s}$. The displacement

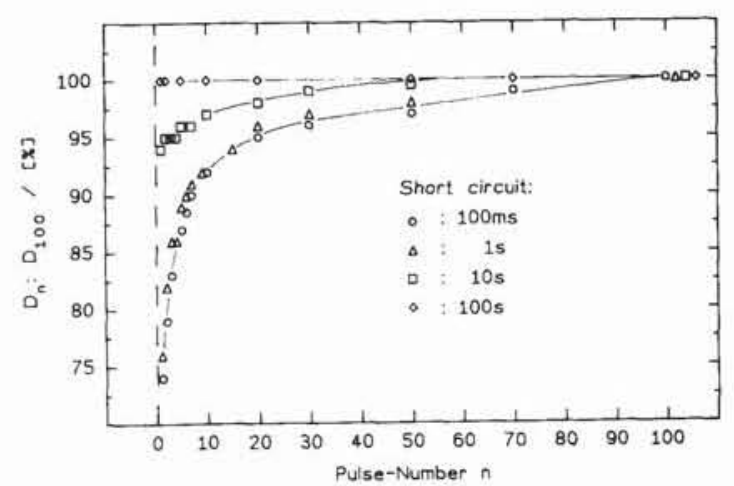

Figure 6 .

Development of the displacement during poling $75 / 25$ mole\% $\mathrm{P}(\mathrm{VDF} / \mathrm{TrFE})$ samples with 100 pulses of $100 \mathrm{~ms}$ pulse length, field strength 80 $\mathrm{MV} / \mathrm{m}$. The values are related to the final value after 100 pulses $\left(0: 47 \mathrm{mC} / \mathrm{m}^{2} ; \triangle: 42 \mathrm{mC} / \mathrm{m}^{2}\right.$; ㅁ: $\left.41 \mathrm{mC} / \mathrm{m}^{2} ; \diamond: 40 \mathrm{mC} / \mathrm{m}^{2}\right)$. Short circuit time between the pulses: o: $100 \mathrm{~ms} ; \Delta: 1 \mathrm{~s} ;$ : $10 \mathrm{~s}$; $\diamond: 100 \mathrm{~s}$

and the remanent polarization grow very fast during the first 20 pulses and slower during the next pulses. But if $t_{a c}$ is increased to 10 and $100 \mathrm{~s}$ there is a change. At $t_{s c}=100 \mathrm{~s}$ the displacement does not grow during the 100 pulses. From pulse to pulse it takes always the same value, in contrast to $t_{s c} \leqslant 1 \mathrm{~s}$ (Figure 6). The remanent polarization also stays almost constant from pulse to pulse (Figure 7). But after applying another 100 pulses to this sample with $t_{s c}=100 \mathrm{~ms}$ the displacement as well as the remanent polarization grow during this second pulse series. Shortening $t_{s c}$ causes an increase of the displacement and the remanent polarization.

These experiments indicate that a critical short circuit time $t_{c r i t}$ between the pulses exists, in which the displacement does not change at all and the remanent polarization increases only a little. The order of magnitude of $t_{c r i t}$ amounts 10 to $100 \mathrm{~s}$ for the $\mathrm{P}(\mathrm{VDF} / \mathrm{TrFE}) 75 / 25$ copolymer. If the short circuit duration is $<t_{\text {crit }}$, for example $1 \mathrm{~s}$, this short circuit time between the pulses does not make a great difference to poling under constant voltage.

On the other hand we can estimate the time $\tau$, which injected charges need to leave the copolymer film under short circuit. This time $\tau$ is given by the dielectric relaxation time $\tau=\epsilon \epsilon_{0} \rho\left(\rho \approx 10^{12} \Omega \mathrm{cm}\right.$ resistivity, $\epsilon=10$ to 15$)$ of $100 \mathrm{~s}$. The agreement between $t_{c r i t}$ and $\tau$ indicates that charges participate in the development of the remanent polarization. 


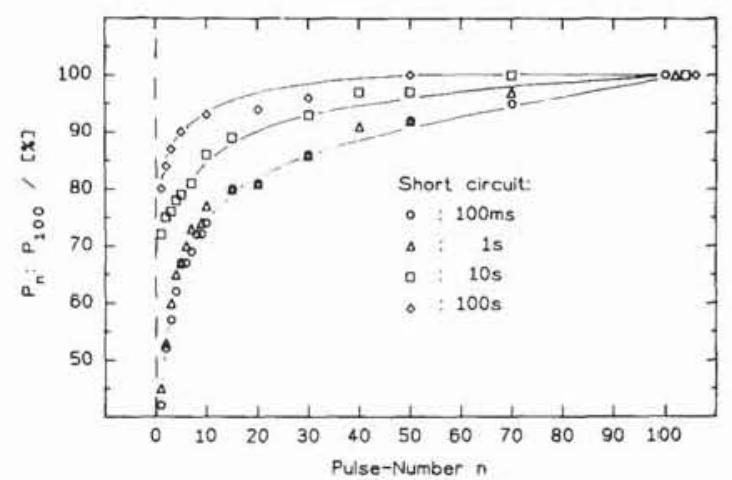

Figure 7.

Development of the remanent polarization during poling $75 / 25$ mole $\% \mathrm{P}(\mathrm{VDF} / \mathrm{TrFE})$ samples with 100 pulses of $100 \mathrm{~ms}$ pulse length, field strength 80 $\mathrm{MV} / \mathrm{m}$. The values are related to the final value after 100 pulses (o: $34 \mathrm{mC} / \mathrm{m}^{2} ; \Delta: 25 \mathrm{mC} / \mathrm{m}^{2}$; : $\left.24 \mathrm{mC} / \mathrm{m}^{2} ; \diamond: 20 \mathrm{mC} / \mathrm{m}^{2}\right)$. Short circuit time between the pulses: $0: 100 \mathrm{~ms} ; \triangle: 1 \mathrm{~s} ; \square: 10 \mathrm{~s}$; $\diamond: 100 \mathrm{~s}$.

\section{DISCUSSION}

$\mathrm{T}$ HE delayed development of the remanent polarization as compared to the fast development of the dielectric displacement can be explained by two mechanisms.

The first mechanism is the orientation of the crystallite dipoles in the field direction under an external electric field. Since the predictions of the $60^{\circ}$ model $[7,8$, 24] agree with the measured development of the displacement under field [12], it appears justified to assume that the dipoles in $75 / 25$ mole\% $\mathrm{P}(\mathrm{VDF} / \mathrm{TrFE})$ and in PVDF crystallites are oriented under fields similar to that in hard ferroelectric materials. But in contrast to hard ferroelectrics our experiments show that the dipole orientation under field alone makes this orientation not remanent after removing the field.

Therefore the $60^{\circ}$ model must be supplemented by a second model which describes the dipoles stabilization by the field of injected charges, which are trapped at the surface of the crystallites $[16,18,23]$. This supplemented model can explain the observed time delay of the remanent polarization compared to the displacement under field.

Charge injection and trapping can also explain why the rise of the remanent polarization in repeated pulse poling experiments depends on the duration of the short circuit time between the pulses. The fact that the dielectric relaxation time $\tau$ agrees with $t_{\text {crit }}$ indicates that injected charges can escape if the short circuit duration approaches $t_{c r i t}$ and are not longer available for the stabilization process of the remanent polarization. In the view of this supplemented model the poling process of unpoled $\mathrm{P}(\mathrm{VDF} / \mathrm{TrFE})$ films can be described as follows.

The crystallite dipoles are oriented by applying an electric field. Simultaneously charges are injected into the polymer film. Both the orientation and the injection, depend on the field strength. After the dipoles are aligned in the field direction, charge trapping at the crystallite surface is possible. If the field is switched off, the dipoles keep their orientation in the field of these trapped charges. All dipoles which are not fixed in the field of trapped charges distribute randomly because of their thermal energy. They do not contribute to the remanent polarization. Vice versa, injected charges which are not trapped leave the film under short circuit condition or recombine.

If an electric field is applied in the reverse direction to prepolarized films, the dipoles can be oriented in the opposite direction and the charges are set free. If the field is removed before all charges are set free and before the inverted dipoles are stabilized by new charges, the remanent polarization points in the old direction, but with a reduced amplitude. After longer poling all dipoles are inverted and fixed again by new charges under field. As consequence of the primary poling there are already charges in polarized films. That is why the inverted remanent polarization grows faster as if unpolarized films are poled (compare Figure 4 with Figure 5).

\section{SUMMARY}

$\mathrm{M}$ EASUREMENTs at unpoled $75 / 25$ mole\% P(VDF/ TrFE) films show that a time delay of the development of remanent polarization compared to the fast development of the displacement under field was observed as in pure PVDF films [16]. This time delay cannot be explained by a ferroelectric model with 6 -side potential minima for the chain rotation in crystallites alone.

Therefore the dipole orientation model was supplemented by a model which describes the dipole stabilization by the field of trapped charges at the surface of the crystallites. The dipoles are fixed in the field of this charge as also suggested in [16]

In poling copolymer films with repeated short pulses the dependence of the development of the displacement and the remanent polarization on the short circuit time between the pulses also indicates that injected charges 
are participating in the development of the remanent polarization.

\section{ACKNOWLEDGEMENT}

We thank Prof. Gerhard Sessler for the fruitful cooperation in the field of piezoelectric polymers during many years. We are very grateful for many enthusiastic and critical discussions on the fundamental physics involved in this material.

\section{REFERENCES}

[1] Kawai, "The Piezoelectricity of Polyvinylidene Fluo ride", Jap. J. Appl. Phys., Vol. 8, pp. 975-976, 1969.

[2] T. Yamada, T. Ueda, T. Kitayama, "Ferroelectricto-paraelectric Phase Transition of Vinylidene Fluoride-trifluoroethylene Copolymer", J. Appl. Phys., Vol. 52, pp. 948-952, 1981.

[3] G. M. Sessler, "Piezoelectricity in Polyvinylidene Fluoride", J. Acoust. Soc. Am., Vol. 70, pp. 1596$1608,1981$.

[4] M. G. Broadhurst, G. T. Davies, "Piezo and Pyroelectric Properties", Chapter 5 in: Sessler G. M. (ed.), Electrets, Springer Berlin, 1980, 1987.

[5] R. G. Kepler, R. A. Anderson, "Piezoelectricity in Polymers", CRC Critical Reviews in Solid State and Materials Science, Vol. 9, pp. 399-447, 1980.

[6] K. Tashiro, K. Takano, M. Kobayashi, Y. Chatani, H. Tadokoro, "Structural Study on Ferroelectric Phase Transition of Vinylidene Fluoridetrifluoroethylene Copolymers (III) Dependence of Transitional Behavior on VDF Content", Ferroelectrics, Vol. 57, pp. 297-326, 1984.

[7] M. G. Broadhurst, G. T. Davies, "Ferroelectric Polarization in Polymers", Ferroelectrics, Vol. 32, pp. 177-180, 1981.

[8] R. G. Kepler, R. A. Anderson, "Ferroelectricity in Polyvinylidene Fluoride", J. Appl. Phys., Vol. 49, pp. 1232-1235, 1978.

[9] K. Holdik, W. Eisenmenger, "Charge and Polarization Dynamics in Polymer Films", in G. M. Sessler (ed.), Proc. 5th Int. IEEE Symp. Electrets, Piscataway, pp. 553-558, 1985.
[10] W. J. Merz, "Domain Formation and Domain Wall Motions in Ferroelectric $\mathrm{BaTiO}_{3}$ Single Crystals", Phys. Rev., Vol. 95, pp. 690-698, 1954.

[11] T. Furukawa, M. Date, G. E. Johnson, "Polarization Reversal Associated with Rotation of Chain Molecules in $\beta$-phase Polyvinylidene Fluoride", J. Appl. Phys., Vol. 54, pp. 1540-1546, 1983.

[12] T. Furukawa, M. Date, M. Ohuchi, A. Chiba, "Ferroelectric Switching Characteristics in a Copolymer of Vinylidene Fluoride and Trifluoroethylene", J. Appl. Phys., Vol. 56, pp. 1481-1486, 1984.

[13] T. Furukawa, H. Matsuzaki, M. Shina, Y. Tajitsu, "Nanosecond Switching in Thin Films of Vinylidene Fluoride/Trifluoroethylene Copolymers", Jap. J. Appl. Phys. Vol. 24, pp. 534-535, 1985.

[14] A. Odajima, T. T. Wang, Y. Takase, "An Explanation of Switching Characteristics in Polymer Ferroelectrics by a Nucleation and Growth Theory", Ferroelectrics, Vol. 62 , pp. $39-46,1985$

[15] Y. Tajitsu, T. Masuda, T. Furukawa, "Switching Phenomena in Vinylidene Fluoride/Trifluoroethylene near the Curie Point”, Jap J. Appl. Phys., Vol. 26, pp. $1749-1753,1987$.

[16] M. Womes, E. Bihler, W. Eisenmenger, "Dynamics of Polarization Growth and Reversal in PVDF Films", IEEE Transactions on Electrical Insulation, Vol. 24, pp. 461-468, 1989.

[17] M. Haardt, W. Eisenmenger, "High Resolution Technique for Measuring Charge and Polarization Distributions in Dielectrics by Piezoelectrically Induced Pressure Step Waves (PPS)", IEEE 1982 Annual Report Conf. Elect. Insul. and Dielect. Phen., pp. 4651, Piscataway 1982.

[18] W. Eisenmenger, M. Haardt, "Observation of Charge Compensated Polarization Zones in PVDF", Solid State Comm., Vol. 41, pp. 917-920, 1982.

[19] Y. Tajitsu, H. Ogura, A. Chiba, T. Furukawa, "Investigation of Switching Characteristics of Vinylidene Fluoride/Trifluoroethylene in Relation to Their Structures", Jap. J. Appl. Phys., Vol. 26, pp. 554$556,1987$.

[20] V. F. Fernandez, A. Suzuki, A. Chiba, "Study of Annealing Effects on the Structure of Vinylidene Fluoride/Trifluoroethylene Copolymers using WAXS and SAXS, Macromolecules, Vol. 20, pp. $1806-1811,1987$. 
[21] K. Koga, H. Ohigashi, "Piezoelectricity and Related Properties of Vinylidene Fluoride and Trifluoroethylene Copolymers", J. Appl. Phys., Vol. 56, pp. 2142$2150,1986$.

[22] M. Jimbo, T. Fukada, H. Takeda, F. Suzuki, K. Horino, K. Koyama, S. Ikeda, Y. Wada, "Ferroelectric Switching Characteristics of $73 / 27$ Copolymer of Vinylidene Fluoride and Trifluoroethylene", J. Polym. Sci., Part B: Polym. Phys., Vol. 24, pp. 909-921, 1986.

[23] W. Eisenmenger, M. Haardt, K. Holdik, "Observation of Charge Compensated Polarization Zones in Polyvinylidenefluoride (PVDF) Piezoelectrically Generated Pressure Step Wave (PPS) Response",
IEEE 1982 Annual Report Conf. Elect. Insul. and Dielect. Phen., pp. 52-57, Piscataway 1982.

[24] H. Dvey-Aharon, T. J. Sluckin, P. L. Taylor, "Kink Propagation as a Model for Poling in Polyvinylidene Fluoride", Phys. Rev. B, Vol. 21, pp. 3700-3707, 1980

[25] J. B. Lando and W. W. Doll, "The Polymorphism of Polyvinylidene Fluoride. I. The Effect of Head-toHead Structure", J. Macromol. Sci.-Phys., Vol. B2, pp. 205-218, 1968

Manuscript was received on 21 May 1990, in revised form 13 Dec 1990. 